\title{
Cosmological and thermodynamics analysis in Weyl gravity
}

\author{
Abdul Jawad ${ }^{\mathrm{a}}$, Zoya Khan ${ }^{\mathrm{b}}$, Shamaila Ranic \\ Department of Mathematics, COMSATS University Islamabad, Lahore-Campus, Lahore 54000, Pakistan
}

Received: 31 August 2019 / Accepted: 3 January 2020 / Published online: 30 January 2020

(c) The Author(s) 2020

\begin{abstract}
In the framework of modified Weyl gravity, we observe the equilibrium picture of the thermodynamical laws for flat Friedmann-Robertson-Walker metric with chameleon scalar field and analyze the validity of the generalized second law of thermodynamics and thermal equilibrium condition for Hubble horizon along with BekenstienHawking entropy. Also, we examine the effective equation of state parameter as well as the square speed of sound. By assuming four different choices of deceleration parameter, we investigate the behavior of equation of state parameter as well as the square speed of sound. The validity of generalized second law of thermodynamics and thermal equilibrium condition is also checked by taking the observational values of the model parameters from $\mathrm{CC}+H_{o}$ dataset.
\end{abstract}

\section{Introduction}

In cosmology, current experimental information appear to show the recent cosmic expansion of the universe. On a broad range, physical cosmology covers the investigation of the description of the universe. From the different recognize cosmological evidence the current cosmic acceleration of the universe disclosed [1-7], which is measured by certain analyses [8-12]. In the present age current perceptions fully recommend that the cosmic acceleration is experiencing by the universe $[13,14]$. The force is generally indicated as dark energy (DE), which is responsible for a development in a specific phase of the universe. To coordinate with later examined data different DE models have been proposed and in this arrangement the most least complex model is the $\Lambda \mathrm{CDM}$ (Cold dark matter) model, which is in great concurrence with the ongoing observational information. In general relativity (GR), this model is obtained by presenting the

\footnotetext{
a e-mails: jawadab181@yahoo.com; abduljawad@cuilahore.edu.pk

be-mail: zoyakhan4814@gmail.com

c e-mail: shamailatoor.math@yahoo.com
}

cosmological constant $\Lambda$, for which equation of state (EoS) parameter is $w_{\Lambda}=-1[15,16]$.

To illustrate the entire development history of the universe, a cosmological model needs both an accelerated and a decelerated phase, for this the deceleration parameter plays a vital part [3,17-21]. A valuable tool against a progressively development history of the universe is the parametrization of the deceleration parameter $q$. Up until now a few wellknown parameterizations for the deceleration parameter have been introduced [22-24]. Mamon and Das [25] presented a logarithmic parameterization of the deceleration parameter in the spatially flat FRW metric. They used type Ia supernovae, cosmic microwave background and baryon acoustic oscillation dataset and also they reconstructed the EoS, deceleration parameter and the jerk parameter compared with reconstructed models of parameterized deceleration parameter with other known parameterizations of $q$. Mamon [26] introduced a generalized parametrization of $q$ to studied the development history of the universe. They used the recent measurement of the Hubble parameter found from the type Ia supernovae and cosmic chronometer model data. Furthermore, the parametric approach likewise improves productivity of the future cosmological overviews. To determine the transition from decelerating phase to an accelerating phase it is reasonable to follow a parametric approach. Inspired by these facts, we have picked a special type of deceleration parameter so that the parameterized deceleration parameter $q(z)$ will give the ideal property for sign flip from a decelerating phase to an accelerating phase.

For the expansion of the universe many theories have represented models, however none of them was totally fruitful. GR was not conformally invariant theory [27], therefore, as an alternative theory of GR modified conformal gravity was proposed [28,29]. Under the scale transformation the conformal Weyl gravity is the primary gravitational theory which is constant. Hermann Weyl [30,31] introduced conformal gravity or Weyl gravity, for cosmology conformal gravity is also of concern. Tanhayi et al. [32] shown that the estimated value 
of cosmological constant and Hubble parameter are close to their measured values. They studied the dependence of Hubble parameter and cosmological constant in Weyl gravity, as a function of $t$ and $r$. Ghanaatian et al. [33] considered the modified gravity coupled by Weyl tensor in spatially flat Friedmann-Lemaître-Robertson-Walker (FLRW) metric, they described the cosmic expansion of the universe also they checked stability conditions of models. Varieschi and Ault [34] analyzed the classic wormhole geometries in conformal Weyl gravity. They described the main energy condition as well as distinct wormhole solutions.

It is generally trusted that, investigations of the associations among thermodynamics and gravity theories would gives us some understanding into the genuine idea of gravity. For this reason generally many models have been examined, specifically gravity theories for example braneworld [35-37], Lovelock and Gauss-Bonnet gravity [38,39], $f(R)$ [40-44] models. Geng et al. [45] verified the generalized first and second law of thermodynamics with the disformal transformation of the $f(R)$ gravity in the FLRW metric. They studied the equilibrium as well as non-equilibrium picture of the generalized second law of thermodynamics (GSLT). Zubair et al. [46] examined the first law of thermodynamics (FLT) and GSLT at apparent horizon in FRW universe in $f\left(R, R_{\alpha \beta} R^{\alpha \beta}, \phi\right)$ gravity, where $R$ is the Ricci invariant, $R_{\alpha \beta} R^{\alpha \beta}$ is the Ricci tensor and $\phi$ is the scalar field respectively. They presented the equilibrium as well as nonequilibrium picture of GSLT and to observed the validity of GSLT they choose some specific models.

This paper is structure as follows. In Sect. 2 we discuss the model of the Weyl gravity and construct the cosmological parameters and define the four different models of Hubble parameter. In Sect. 3 we examine the equilibrium picture of thermodynamics and analyze the GSLT. In Sect. 4 we define the observational values from the dataset and observe the behavior of cosmological parameters, validity of GSLT as well as thermal equilibrium condition and in Sect. 5 we conclude our result.

\section{Basics of Weyl gravity}

The action [47] for Weyl gravity is given as

$$
\begin{aligned}
S= & -\int \sqrt{-g} d^{4} x\left[\frac{C_{\mu \nu \rho \lambda} C^{\mu \nu \rho \lambda}}{k}\right. \\
& \left.+\frac{g^{\mu \nu} \partial_{\mu} \phi \partial_{\nu} \phi}{2}-V(\phi)-f(\phi) \mathcal{L}_{m}\right],
\end{aligned}
$$

where $\mathcal{L}_{m}$ is the matter Lagrangian density, $V(\phi)$ is the potential term dependent on chameleon scalar field $\phi, f(\phi)$ is an analytical function which is the modification of matter
Lagrangian density and $C_{\mu \nu \rho \lambda}$ is the Weyl tensor, which is given as

$$
\begin{aligned}
C_{\mu \nu \rho \lambda}= & R_{\mu \nu \lambda \rho}-\frac{1}{2}\left(g_{\mu \lambda} R_{\nu \rho}-g_{\mu \rho} R_{\nu \lambda}-g_{\nu \lambda} R_{\mu \rho}+g_{\nu \rho} R_{\mu \lambda}\right) \\
& +\frac{R}{6}\left(g_{\mu \lambda} g_{\nu \rho}-g_{\mu \rho} g_{\nu \lambda}\right) .
\end{aligned}
$$

Putting the Weyl tensor in Eq. (1), we get

$$
\begin{aligned}
S= & -\int \sqrt{-g} d^{4} x\left[\frac{1}{k}\left(R^{\mu \nu \rho \lambda} R_{\mu \nu \rho \lambda}-2 R^{\mu \nu} R_{\mu \nu}+\frac{R^{2}}{3}\right)\right. \\
& \left.+\frac{g^{\mu \nu} \partial_{\mu} \phi \partial_{\nu} \phi}{2}-V(\phi)-f(\phi) \mathcal{L}_{m}\right]
\end{aligned}
$$

the term $\sqrt{-g}\left(R^{\mu \nu \rho \lambda} R_{\mu \nu \rho \lambda}-4 R^{\mu \nu}+R^{2}\right)$ is a Gauss-Bonnet term and during the integration of Eq. (3) it disappears. The term $R^{\mu \nu \rho \lambda} R_{\mu \nu \rho \lambda}$ can be written as $R^{2}$ and $R^{\mu \nu} R_{\mu \nu}$. Then the action is given as

$$
\begin{aligned}
S= & -\int \sqrt{-g} d^{4} x\left[\frac{2}{k}\left(R_{\mu \nu} R^{\mu \nu}-\frac{R^{2}}{3}\right)\right. \\
& \left.+\frac{g^{\mu \nu} \partial_{\mu} \phi \partial_{\nu} \phi}{2}-V(\phi)-f(\phi) \mathcal{L}_{m}\right] .
\end{aligned}
$$

By solving the Eq. (4) about the metric $g^{\mu \nu}$, the field equation $[48,49]$ is define as

$f T_{\mu \nu}^{(m)}+T_{\mu \nu}^{(\phi)}=\frac{4 \mathcal{W}_{\mu \nu}}{k}$,

where $T_{\mu \nu}^{(\phi)}$ is the energy-momentum tensor and $T_{\mu \nu}^{(m)}$ is the energy-momentum tensor in the form of perfect fluid of the non-relativistic matter and

$$
\begin{aligned}
\mathcal{W}_{\mu \nu}= & -\frac{g_{\mu \nu} \square R}{2}-\square R_{\mu \nu}+\nabla_{\rho} \nabla_{\mu} R_{\nu}^{\rho} \\
& +\nabla_{\rho} \nabla_{\nu} R_{\mu}^{\rho}-2 R_{\mu}^{\rho} R_{\nu} \rho \\
& +\frac{g_{\mu \nu} R_{\rho \lambda} R^{\rho \lambda}}{2}-\frac{2 \nabla_{\mu} \nabla_{\nu} R}{3}+\frac{2 g_{\mu \nu} \square R}{3} \\
& +\frac{2 R R_{\mu \nu}}{3}-\frac{g_{\mu \nu} R^{2}}{6}, \\
T_{\mu \nu}^{(\phi)}= & \partial_{\mu} \phi \partial_{\nu} \phi-g_{\mu \nu}\left(\frac{g^{\alpha \beta} \partial_{\alpha} \phi \partial_{\beta} \phi}{2}-V(\phi)\right), \\
T_{\mu \nu}^{(m)}= & g_{\mu \nu} \mathcal{L}_{m}-\frac{2 \Delta \mathcal{L}_{m}}{\Delta g^{\mu \nu}},
\end{aligned}
$$

and $f=f(\phi)$ and $R_{\mu \nu}$ is the Ricci tensor.

Now, the flat FRW metric is given by

$d s^{2}=d t^{2}-a(t)^{2}\left[d r^{2}+r^{2}\left(d \theta^{2}+\sin \theta^{2} d \varphi^{2}\right)\right]$,

from Eq. (9) the standard modified FRW equations defined as

$$
\begin{aligned}
3 H^{2} & =\rho_{e f f}, \\
\dot{H} & =-\frac{1}{2}\left(\rho_{e f f}+p_{e f f}\right),
\end{aligned}
$$


where $H \equiv \frac{\dot{a}}{a}$ is the expansion rate with respect to the cosmic time, $\rho_{e f f}$ and $p_{e f f}$ are effective energy density and pressure of the system, here $\rho_{\text {eff }}$ and $p_{\text {eff }}$ are

$$
\begin{aligned}
& \rho_{e f f}=\rho_{R}+\rho_{m} f, \\
& p_{e f f}=p_{R}+\gamma \rho_{m} f,
\end{aligned}
$$

where $\gamma=\frac{p_{m}}{\rho_{m}}$ and

$$
\begin{aligned}
\rho_{R}= & V(\phi)+\frac{\dot{\phi}^{2}}{2}+\frac{3 H^{2}}{k}-\frac{48 H \ddot{H}}{k} \\
p_{R}= & V(\phi)-\frac{\dot{\phi}^{2}}{2}-\frac{3 H^{2}}{k}-\frac{2 \dot{H}}{k}-\frac{72}{k}\left(5 H^{4}+\frac{26 \dot{H} H^{2}}{3}\right. \\
& \left.+\frac{4 H \ddot{H}}{3}+\dot{H}^{2}\right) .
\end{aligned}
$$

The energy conservation equations are given as

$$
\begin{aligned}
& \dot{\rho}_{m}+3 H \rho_{m}(1+\gamma)=-\frac{\rho_{m} \dot{f}}{f}, \\
& \dot{\rho}_{R}+3 H \rho_{R}(1+w)=0 .
\end{aligned}
$$

From action (4) the wave equation of motion for chameleon scalar field is obtained as

$\ddot{\phi}+3 H \dot{\phi}+V_{\phi}+\gamma \rho_{m} f_{\phi}=0$,

where subscript $\phi$ denotes the derivative with respect to scalar field. Now from (13), we find

$\rho_{m}=\frac{A}{a^{3(1+\gamma)} f}$,

where A is the constant term. By using Eqs. (11) and (12) the effective EoS is define as

$$
\begin{aligned}
\frac{d \rho_{e f f}}{d t}= & -3 A H \gamma(1+\gamma) a^{-3(1+\gamma)}+V_{\phi} \dot{\phi}+\dot{\phi} \ddot{\phi} \\
& +6 H \dot{H}-48[H \dddot{H}+\dot{H} \ddot{H}],
\end{aligned}
$$

the square speed of sound tell us about the stability of DE models, the model is stable if $v_{s}^{2}>0$ otherwise it is unstable. The deceleration parameter is define as

$q(z)=-1-\frac{\dot{H}}{H^{2}}=-1+\frac{\left(H^{\prime}(z)\right)^{2}(1+z)}{2 H^{2}(z)}$.

To representing the nature of the universe expansion rate parametrization of the $q$ (deceleration parameter) plays a vital role. For this we choose four different models of parameterized deceleration parameter and find Hubble parameter in terms of redshift.

\section{Model 1:}

The parametrization of this model [25] is given as

$q(z)=q_{0}+q_{1}\left(\frac{\ln (N+z)}{1+z}-\ln N\right) \cdot N>1$

We deal with two parameters $q_{0}, q_{1}$ with their convenient physical analysis, by using the observational data we can efficiently constrain them, so the Eq. (eq21) can clarify the current universe evolution more accurately [25]. By comparing the Eqs. (20) and (21), the Hubble parameter is given by

$H(z)=H_{0} N^{\frac{N q_{1}}{-1+N}}(N+z)^{-\frac{(N+z) q_{1}}{(1+z)(-1+N)}}(1+z)^{\left(1+q_{0}+\frac{q_{1}}{-1+N}-q_{1} \ln N\right)}$,

from the parameter of EoS we classify the acceleration and deceleration aspects of the universe. By taking the time derivative of Eq. (11) the square speed of sound is given as

$v_{s}^{2}=\frac{\frac{d p_{e f f}}{d t}}{\frac{d \rho_{e f f}}{d t}}$,

where

$$
\begin{aligned}
\frac{d p_{e f f}}{d t}= & -3 A H \gamma(1+\gamma) a^{-3(1+\gamma)}+V_{\phi} \dot{\phi}-\dot{\phi} \ddot{\phi} \\
& -6 H \dot{H}-2 \ddot{H}-72\left[4 \dot{H} H^{3}\right. \\
& \left.+\frac{26}{3}\left[2 \dot{H}^{2} H+\ddot{H} H^{2}\right]+\frac{4}{3}[\dot{H} \ddot{H}+\dddot{H} H]+2 \dot{H} \ddot{H}\right],
\end{aligned}
$$

where $q_{0}, q_{1}, N$ are model parameters and $H_{0}$ is the current value of Hubble parameter, which is describe as $H_{0}=67$.

Model 2:

The linear parametrization of the deceleration parameter is given by [3]

$q(z)=q_{0}+q_{1} z$.

The above linear deceleration parameter (which is linear in $z$ (cosmic redshift) and $a$ (scale factor)) utilized to examine the kinematics of the universe [50]. By taking Eqs. (20) and (23) the Hubble parameter is derived as

$H(z)=H_{0} e^{q_{1} z}(1+z)^{\left(1+q_{0}-q_{1}\right)}$. 
Model 3:

Next parametrization of the $q$ is of following the form [21, 51-55]

$q(z)=q_{0}+\frac{q_{1} z}{1+z}$.

The motivation of this parametric structure for deceleration parameter $q(z)$ comes from one of the most prominent parametrization of the dark energy EoS [56,57] and despite extremely basic, is by all accounts adaptable enough to mimic the $q(z)$ behavior of immense class of acceleration models [58].

The expression of $H(z)$ is evolve for this model as

$H(z)=H_{0} e^{-\frac{q_{1} z}{1+z}}(1+z)^{\left(1+q_{0}+q_{1}\right)}$.

Model 4:

Now we have $q(z)$ for flat $\Lambda$ CDM model [25] which is obtain as

$q(z)=-1+\frac{3}{2\left[1+\frac{\Omega_{\Lambda_{0}}}{\Omega_{m_{0}}}(1+z)^{-3}\right]}$,

where $\Omega_{m_{0}}+\Omega_{\Lambda_{0}}=1$. We use this model to observe the decelerated and accelerated phase of the universe for the best fit values from the observational data. And we chose $\Omega_{m_{0}}=$ 0.375 for flat universe. For this model Hubble parameter is define as

$H(z)=H_{0}\left[\Omega_{m_{0}}(1+z)^{3}+\left(1-\Omega_{m_{0}}\right)\right]^{\frac{1}{2}}$.

\section{Thermodynamics laws in equilibrium characterization}

In this section, we analyze the FLT in modified Weyl gravity at Hubble horizon for flat FRW universe. From the condition $h^{\mu \nu} \partial_{\mu} R_{A} \partial_{\nu} R_{A}=0$ [59-61], Hubble horizon is given as

$R_{A}=\frac{1}{H}$.

By taking the time derivative of Hubble horizon, we have

$d R_{A}=\frac{H R_{A}^{3}}{2}\left(\rho_{e f f}+p_{e f f}\right) d t$

the general Bekenstein-Hawking entropy is defined as $S=$ $\frac{A}{4 G}$, where $A=4 \pi r^{2}$ is the area of the horizon $[62,63]$.

Utilizing the entropy, Eq. (29) becomes

$\frac{G}{2 \pi R_{A}} d S=\frac{H R_{A}^{3}}{2}\left(\rho_{e f f}+p_{e f f}\right) d t$.
The horizon temperature [64] is described as

$$
\begin{aligned}
T_{h}=\frac{\left|k_{s g}\right|}{2 \pi}, \quad k_{s g} & =\frac{1}{2 \sqrt{-h}} \partial_{\mu}\left(\sqrt{-h} h^{\mu \nu} \partial_{\nu} R_{A}\right) \\
& =-\frac{1}{R_{A}}\left(1-\frac{\dot{R}_{A}}{2 H R_{A}}\right)=-\frac{R_{A}}{2}\left(2 H^{2}+\dot{H}\right) .
\end{aligned}
$$

Multiplying $T_{h}=-\frac{1}{2 \pi R_{A}}\left(1-\frac{\dot{R_{A}}}{2 H R_{A}}\right)$ on both sides of Eq. (30), we can get

$T_{h} d S=\left[4 \pi H R_{A}^{3} d t-2 \pi R_{A}^{2} d R_{A}\right]\left(\rho_{e f f}+p_{e f f}\right)$.

The Misner-sharp energy is given as $E=\frac{R_{A}}{4 G}$, the energy density in terms of the volume represent as $V=\frac{4 \pi R_{A}^{3}}{3}$, which is interpret as

$\tilde{E}=\frac{3 H^{2}}{8 \pi G} V \equiv \rho_{e f f} V$.

Taking the differential of Eq. (33), we easily find

$d \tilde{E}=-4 \pi H R_{A}^{3}\left(\rho_{e f f}+p_{e f f}\right) d t+4 \pi R_{A}^{2} \rho_{e f f} d R_{A}$.

Combining the Eqs. (32) and (34), we can acquire

$T_{h} d S=-d \tilde{E}+2 \pi R_{A}^{2}\left(\rho_{e f f}-p_{e f f}\right) d R_{A}$

The work density is described as

$\tilde{W}=-\frac{1}{2}\left(T^{(M) \mu v} h_{\mu \nu}+\tilde{T}^{(d e) \mu v} h_{\mu \nu}\right)=\frac{1}{2}\left(\rho_{e f f}-p_{e f f}\right)$,

using the work density in Eq. (35), we get

$T_{h} d S=-d \tilde{E}+\tilde{W} d V$.

\subsection{Generalized second law of thermodynamics}

To describe the equilibrium picture of the GSLT [46], we can write the Gibbs equation in terms of the density and total pressure which is define as

$T d S_{i}=d\left(\rho_{e f f} V\right)+p_{e f f} d V$,

by taking the time derivative of Eqs. (37) and (38) it leads to

$\dot{S}+\dot{S}_{i}=-\frac{48 \pi^{2} \dot{H}^{2}}{R H^{3}}\left(\rho_{e f f}+p_{e f f}\right)$,

where $R=6\left(\dot{H}+2 H^{2}\right), \quad \dot{S}$ and $\dot{S}_{i}$ represents the horizon entropy and the sum of all entropy components inside the horizon. The condition which satisfy the entropy relation is define as

$\dot{S}_{\text {tot }}=\dot{S}+\dot{S}_{i} \geq 0$ 
From Eq. (39), we can easily get

$\dot{S}_{\text {tot }}=\frac{12 \pi \dot{H}^{2}}{G R H^{3}} \geq 0$.

\section{Power-law correction}

By the power-law correction we choose $\phi=\phi_{o} a^{n}$ and $V(\phi)=\phi^{p}$. By putting the power-law terms the EoS parameter, reads

$$
\begin{aligned}
w_{e f f}= & {\left[A \gamma a^{-3(1+\gamma)}+\left(\phi_{o} a^{n}\right)^{p}-\frac{\left(n \dot{a} a^{n-1} \phi\right)^{2}}{2}-3 H^{2}\right.} \\
& -2 \dot{H}-72\left(H^{4}+\frac{26 \dot{H} H^{2}}{3}+\frac{4 H \ddot{H}}{3}\right. \\
& \left.\left.+\dot{H}^{2}\right)\right]\left[A a^{-3(1+\gamma)}+\left(\phi_{o} a^{n}\right)^{p}+\frac{\left(n \dot{a} a^{n-1} \phi\right)^{2}}{2}\right. \\
& \left.+3 H^{2}-48 H \ddot{H}\right]^{-1} .
\end{aligned}
$$

Inserting the power-law terms in Eq. (18) becomes

$$
\begin{aligned}
v_{s}^{2}= & {\left[-3 H \gamma A(1+\gamma) a^{-3(1+\gamma)}\right.} \\
& +p\left(\phi_{o} a^{n}\right)^{p-1}\left(n \dot{a} a^{n-1} \phi\right)^{2}-n^{2}(n-1) a^{2 n-3} \dot{a}^{3} \phi_{o}^{2} \\
& -n^{2} a^{2 n-2} \dot{a} \ddot{a} \phi_{o}^{2}-6 H \dot{H}-2 \ddot{H} \\
& -72\left(20 \dot{H} H^{3}+\frac{26}{3}\left(2 H \dot{H}^{2}+\ddot{H} H^{2}\right)\right. \\
& \left.\left.+\frac{4}{3}(H \dddot{H}+\ddot{H} \dot{H})+2 \dot{H} \ddot{H}\right)\right] \\
& *\left[-3 H A \gamma(1+\gamma) a^{-3(1+\gamma)}+p\left(\phi_{o} a^{n}\right)^{p-1}\right. \\
& *\left(n \dot{a} a^{n-1} \phi\right)^{2}-n^{2}(n-1) a^{2 n-3} \dot{a}^{3} \phi_{o}^{2} \\
& -n^{2} a^{2 n-2} \ddot{a} \ddot{a} \phi_{o}^{2}-6 H \dot{H} \\
& -48(H \dddot{H}+\dot{H} \ddot{H})]^{-1} .
\end{aligned}
$$

From the power-law terms Eq. (39) can be written as

$$
\begin{aligned}
\dot{S}+\dot{S}_{i}= & -\frac{48 \pi^{2} \dot{H}^{2}}{R H^{3}}\left[\left(A a^{-3(1+\gamma)}+\left(\phi_{o} a^{n}\right)^{p}\right.\right. \\
& +\frac{\left(n \dot{a} a^{n-1} \phi\right)^{2}}{2}+3 H^{2} \\
& -48 H \ddot{H})+\left(A \gamma a^{-3(1+\gamma)}+\left(\phi_{o} a^{n}\right)^{p}\right. \\
& -\frac{\left(n \dot{a} a^{n-1} \phi\right)^{2}}{2}-3 H^{2}-2 \dot{H} \\
& \left.\left.-72\left(H^{4}+\frac{26 \dot{H} H^{2}}{3}+\frac{4 H \ddot{H}}{3}+\dot{H}^{2}\right)\right)\right] .
\end{aligned}
$$

Now, by using four different models of Hubble parameter we observe the validity of GSLT and thermal equilibrium condi-
Table 1 For $q_{o}, q_{1}$ the best fit values from $\mathrm{CC}+H_{o}$ dataset

\begin{tabular}{lll}
\hline$q_{o}$ & $q_{1}$ & In $1 \sigma$ C.L. constraints on $q_{o}, q_{1}$ \\
\hline-0.39 & 0.40 & $-0.56 \leq q_{0} \leq-0.20,0.39 \leq \mathrm{q} 1 \leq 1.74$ \\
-0.41 & 0.54 & $-0.60 \leq q_{0} \leq-0.21,0.18 \leq \mathrm{q} 1 \leq 0.86$ \\
-0.48 & 0.70 & $-0.70 \leq q_{0} \leq-0.24,0.24 \leq \mathrm{q} 1 \leq 1.08$ \\
\hline
\end{tabular}

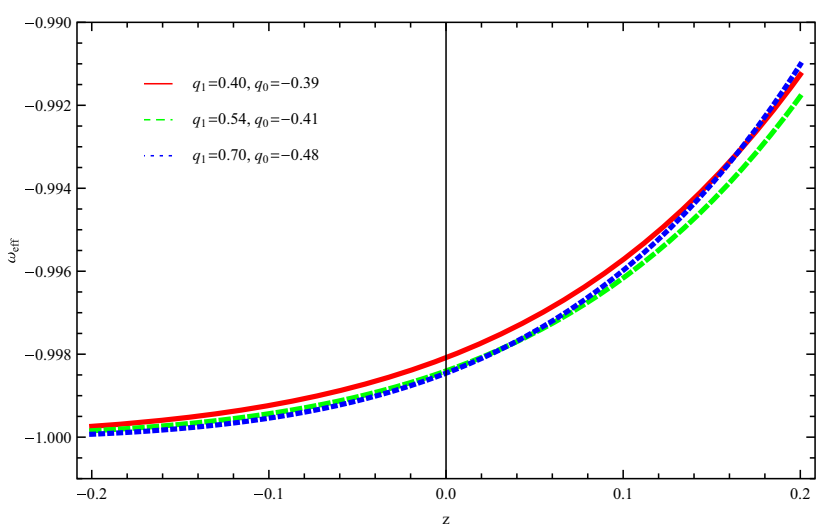

Fig. 1 Plot of $w_{\text {eff }}$ versus $z$ at Hubble horizon with Bekenstein entropy

tion. We also check the stability of the system and decelerated phase of EoS parameter. For this purpose, we consider the observational values of $q_{0}, q_{1}$ and $H_{0}$.

\section{Observational results}

In this section, we express the current Observational dataset and explain the results. We use the observational values from cosmic chronometer (CC) dataset and local value of the Hubble parameter [26]. To measure the Hubble parameter in terms of redshift $\mathrm{CC}$ was first established by Jimenez and Loeb [65]. By using $\mathrm{CC}+\mathrm{H}_{0}$ dataset for all models, graphically we observe the stability of GSLT, thermal equilibrium condition, EoS and square speed of sound. The observational values of the model parameter $\left(q_{o}, q_{1}\right)$ from $\mathrm{CC}+H_{o}$ dataset is given in Table 1 .

\subsection{Model 1}

We plot graphs for model 1 at Hubble horizon with Bekenstein-Hawking entropy for the best fit values of $q_{0}, q_{1}$ from the $\mathrm{CC}+H_{o}$ dataset by taking the values of parameter $A=1.5, \phi=6000, \gamma=-0.3, p=0.1, n=3, N=2$ and the observational value of Hubble constant is $H_{o}=67$.

Figure 1 demonstrate that with the observational values of $q_{o}, q_{1}$ and $H_{o}$ the trajectories of Eos parameter remains in quintessence region at early as well as present epoch and it leads to vacuum DE region at later epoch. In Fig. 2 with 


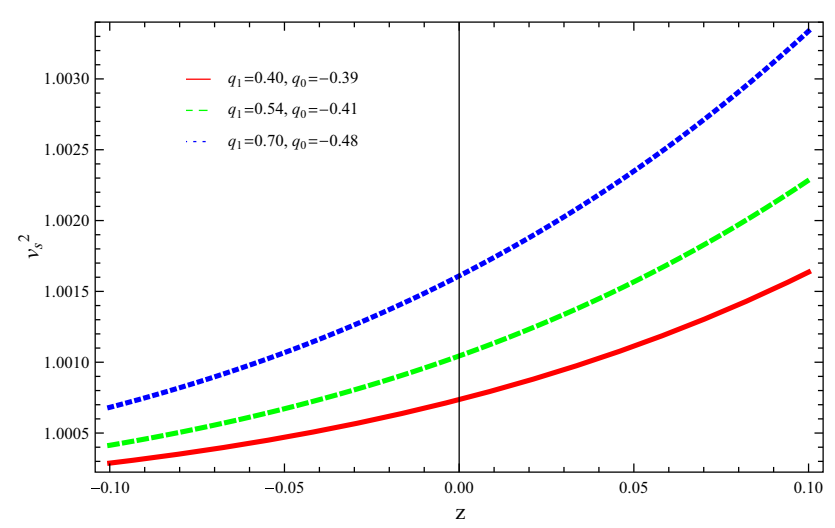

Fig. 2 Plot of $v_{s}^{2}$ versus $z$ at Hubble horizon with Bekenstein entropy

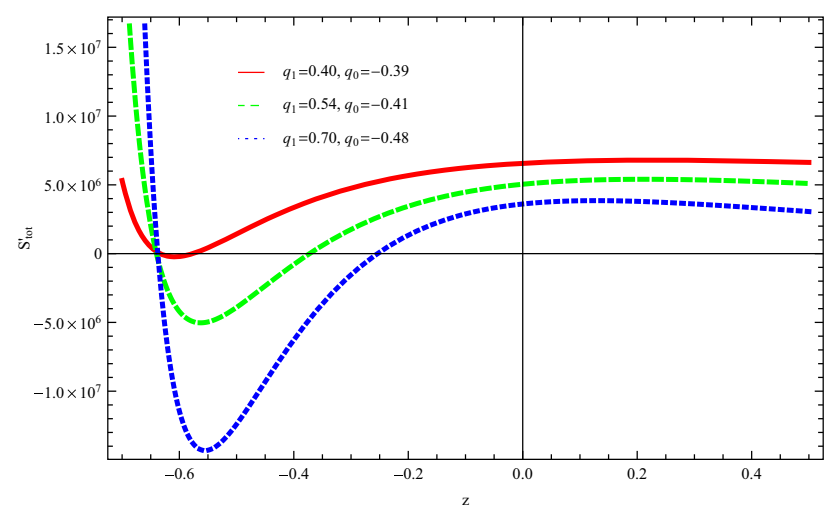

Fig. 3 Plot of $S_{t o t}^{\prime}$ versus $z$ at Hubble horizon with Bekenstein entropy

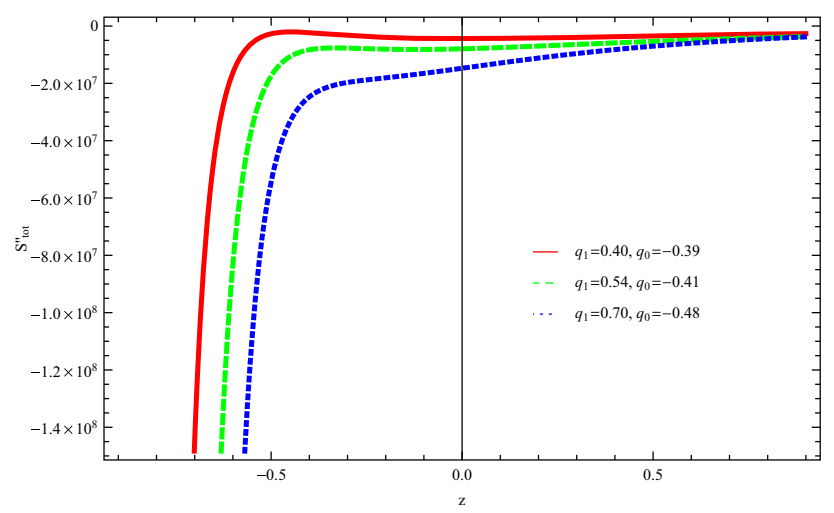

Fig. 4 Plot of $S_{t o t}^{\prime \prime}$ versus $z$ at Hubble horizon with Bekenstein entropy

the same observational values of $q_{o}, q_{1}$ and $H_{o}$ trajectories remains in positive direction at early, present and later epoch which confirms the stability condition i.e. $v_{s}^{2} \geq 0$.

The trajectories in Fig. 3 remains in positive direction at early and present epoch and for $z \geq-0.6$ increasing towards positive direction at later epoch which shows that GSLT is valid at early as well as present epoch and at later epoch GSLT only valid for $z \geq-0.6$. Figure 4 confirms that the

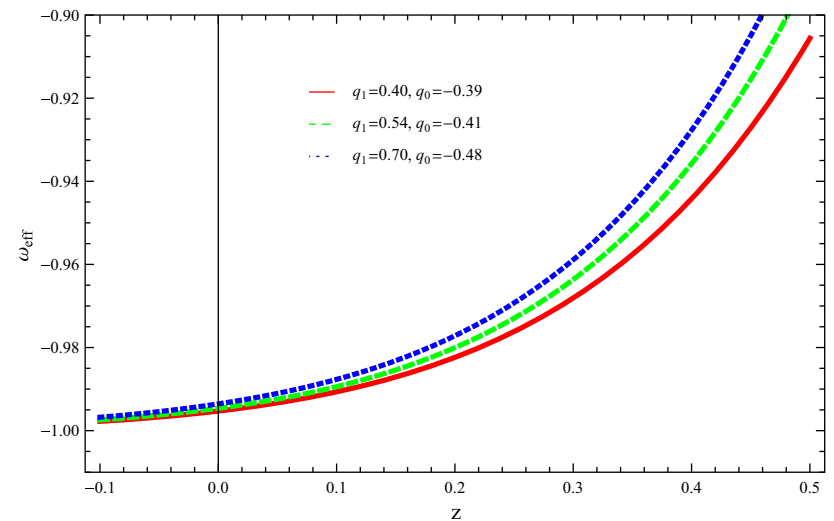

Fig. 5 Plot of $w_{e f f}$ versus $z$ at Hubble horizon with Bekenstein entropy

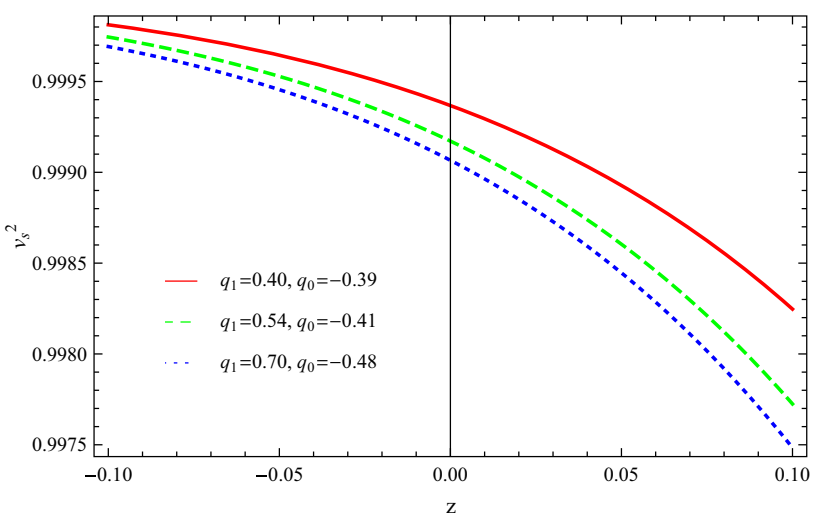

Fig. 6 Plot of $v_{s}^{2}$ versus $z$ at Hubble horizon with Bekenstein entropy

thermal equilibrium condition satisfies at early, present as well as later epoch.

\subsection{Model 2}

By taking the same values of all parameters we plot the graphs and observe the stability of EoS, square speed of sound, GSLT and thermal condition for the best fit values from $\mathrm{CC}+H_{o}$ dataset..

At early as well as present epoch the Eos parameter remains in the quintessence region with the observational values of $q_{o}, q_{1}$ and $H_{o}$ and at later epoch it meet the vacuum DE region in Fig. 5. All trajectories in Fig. 6 increasing towards positive direction which shows the stability for the model 1 at Hubble horizon for the best fit values from observational dataset.

The validity of GSLT confirms in the left side figure for all the observational values as all trajectories gradually decreasing in positive direction at early, present as well as later epoch Fig. 7. The thermal equilibrium condition Fig. 8 cannot satisfies at early as well as present epoch but at later epoch blue trajectory fulfills the thermal condition at $z \geq-0.01$, red 


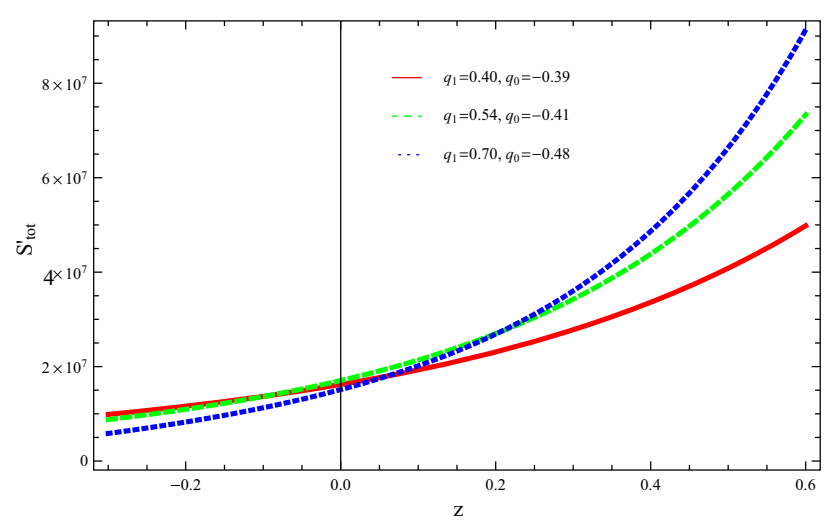

Fig. 7 Plot of $S_{t o t}^{\prime}$ versus $z$ at Hubble horizon with Bekenstein entropy

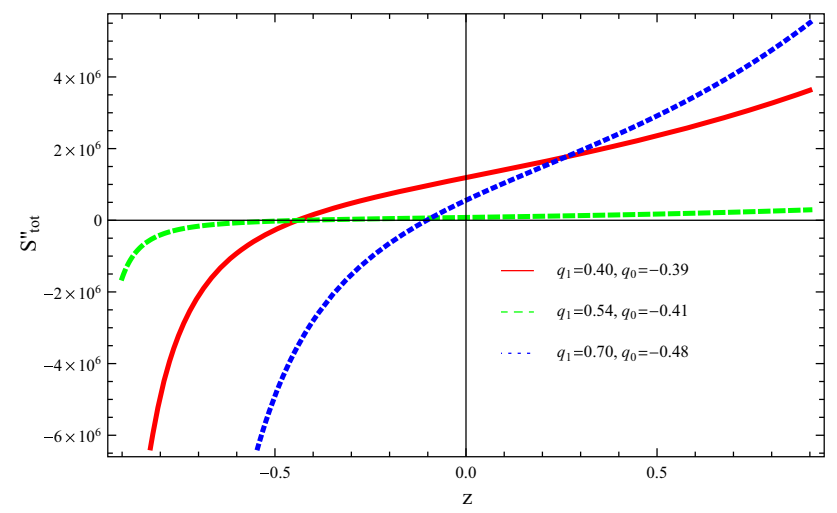

Fig. 8 Plot of $S_{t o t}^{\prime \prime}$ versus $z$ at Hubble horizon with Bekenstein entropy

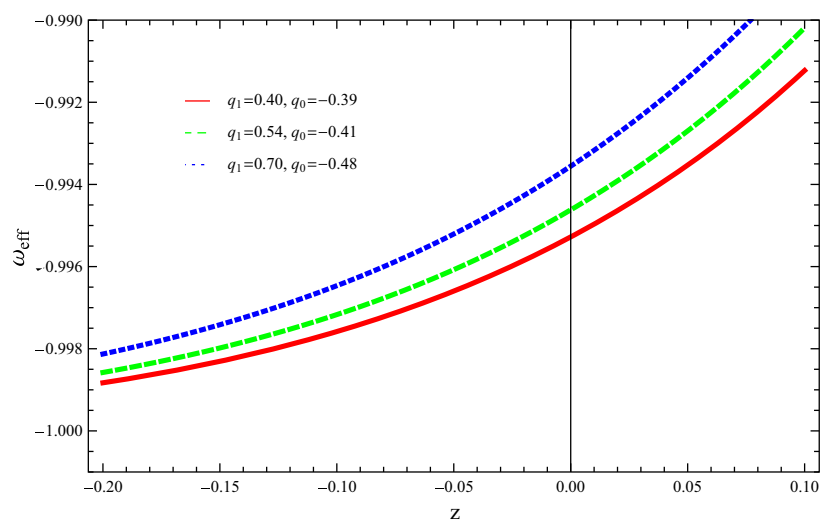

Fig. 9 Plot of $w_{\text {eff }}$ versus $z$ at Hubble horizon with Bekenstein entropy

and green trajectory confirms the thermal equilibrium condition at $z \geq-0.2$. Thus the thermal equilibrium condition satisfies for $z \geq-0.01$ (blue trajectory) and for $z \geq-0.2$ (red and green trajectory) at Hubble horizon.

\subsection{Model 3}

From the $\mathrm{CC}+\mathrm{H}_{0}$ dataset we plot graphs for best fit values with the same values of parameter $A, \phi, p, n, \gamma, n$.

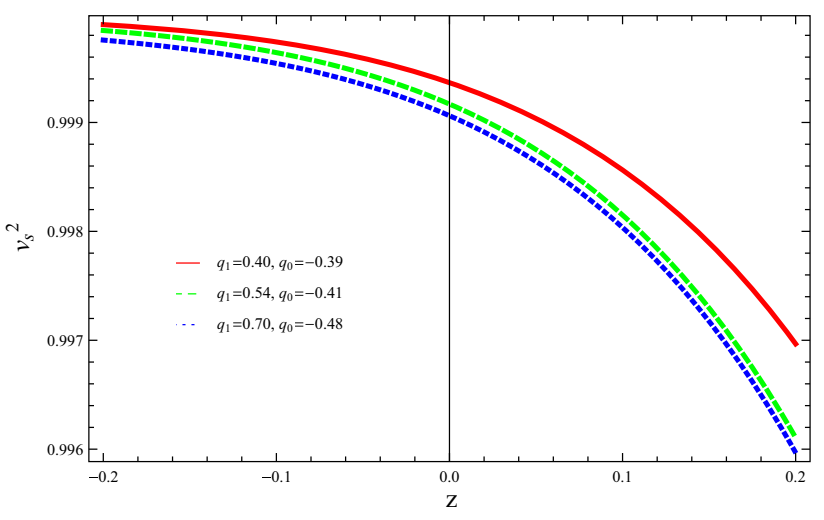

Fig. 10 Plot of $v_{s}^{2}$ versus $z$ at Hubble horizon with Bekenstein entropy

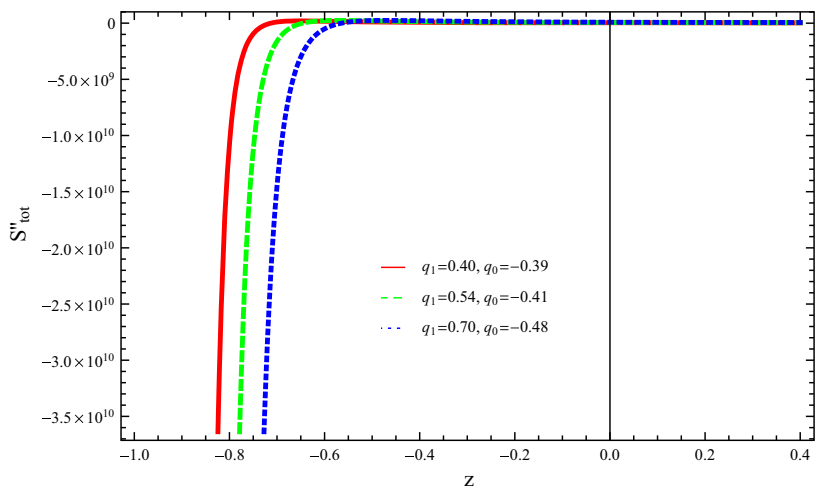

Fig. 11 Plot of $S_{\text {tot }}^{\prime}$ versus $z$ at Hubble horizon with Bekenstein entropy

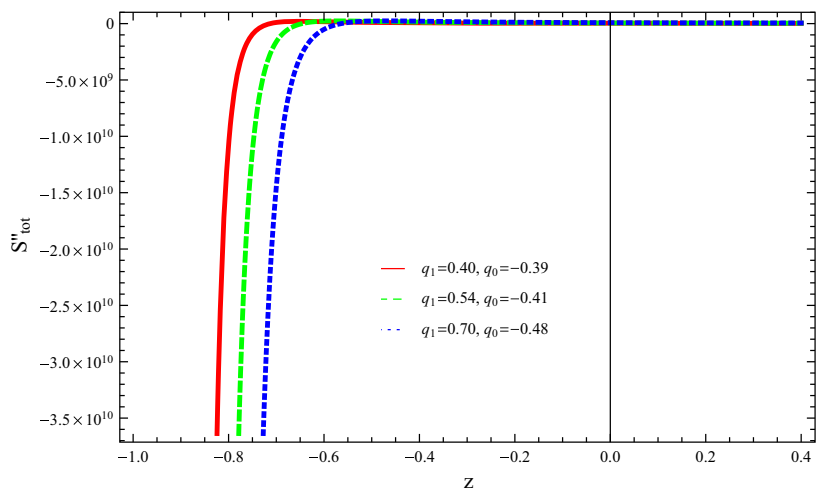

Fig. 12 Plot of $S_{t o t}^{\prime \prime}$ versus $z$ at Hubble horizon with Bekenstein entropy

In Fig. 9 EoS parameter displays the quintessence region in early, present as well as later epoch. The right side trajectories Fig. 10 increasing positively which confirms the stability condition at early, present as well as later epoch.

Figure 11 demonstrate the validity of GSLT at early, present as well as later epoch as all trajectories fulfill the condition $S_{\text {tot }}^{\prime} \geq 0$. All trajectories (Fig. 12) shows that the thermal equilibrium condition satisfies at early, present as well as later epoch. 


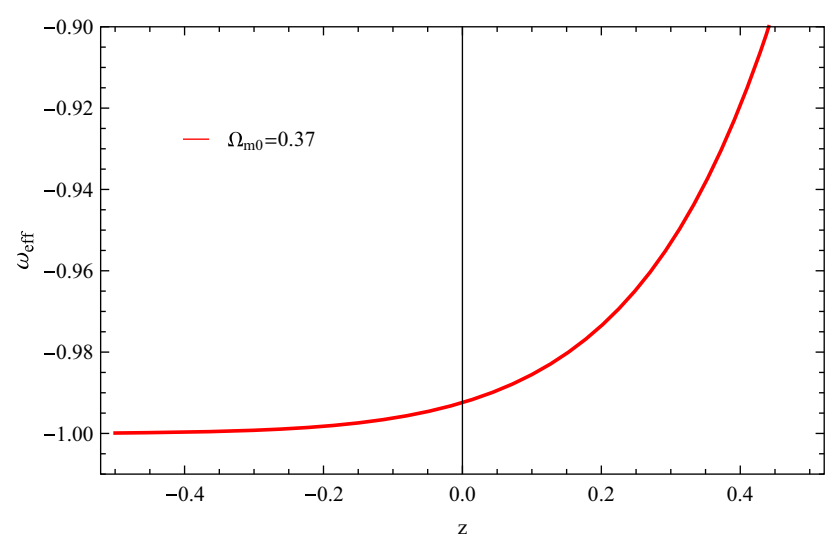

Fig. 13 Plot of $w_{\text {eff }}$ versus $z$ at Hubble horizon with Bekenstein entropy

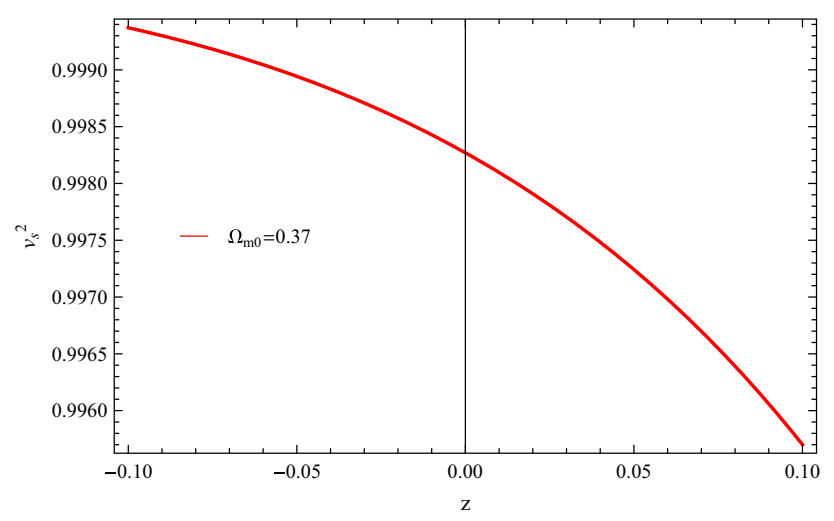

Fig. 14 Plot of $v_{s}^{2}$ versus $z$ at Hubble horizon with Bekenstein entropy

\subsection{Model 4}

In this model we choose the value of $\Omega_{m 0}=0.37$ for flat universe [66] with the same values of all parameters and discuss result at Hubble horizon along with Bekenstein-Hawking entropy.

At early as well as present epoch the EoS parameter remains in quintessence region and at later epoch it gradually decreasing towards the vacuum DE region Fig. 13. The right side trajectory Fig. 14 fulfills the stability condition $v_{s}^{2} \geq 0$ at early, present as well as later epoch.

GSLT is valid at early, present as well as later epoch in Fig. 15 as the condition $S_{\text {tot }}^{\prime} \geq 0$ fulfills. The condition of thermal equilibrium satisfies at early as well as present epoch and at later epoch thermal condition cannot satisfies as the trajectory increasing towards positive phase in Fig. 16.

\section{Summary}

In this work, we observed the validity of GSLT and thermal equilibrium condition in Weyl gravity in a flat FRW uni-

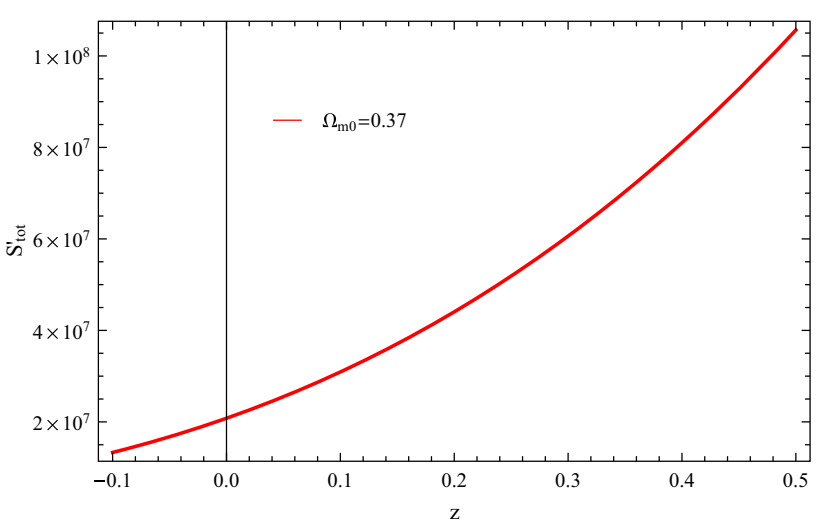

Fig. 15 Plot of $S_{\text {tot }}^{\prime}$ versus $z$ at Hubble horizon with Bekenstein entropy

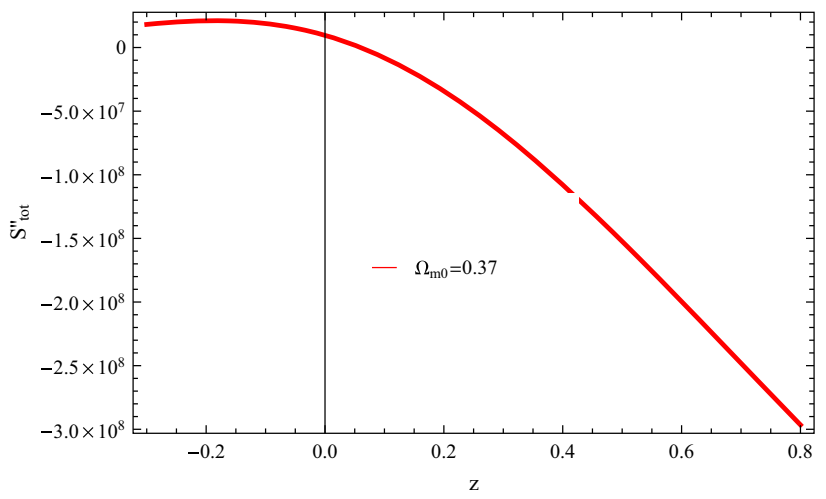

Fig. 16 Plot of $S_{t o t}^{\prime \prime}$ versus $z$ at Hubble horizon with Bekenstein entropy

verse with chameleon scalar field at Hubble horizon with Bekenstein-Hawking entropy, also we constructed the cosmological parameters. We choose different parameterized deceleration parameter and Hubble parameter models in term of redshift, by using these models of Hubble parameter we discussed the validity of GSLT, thermal equilibrium condition as well as we discussed the stability condition and the behavior of EoS parameter. We explored the behavior of GSLT, thermal equilibrium condition and cosmological parameters by using power-law corrections, in terms of redshift. We graphically plot these constructed models and discussed our results for observational values from $\mathrm{CC}+H_{O}$ dataset. We also discussed the behavior of constructed models for the $\triangle \mathrm{CDM}$ model by taking the observational value for flat universe. We used the current measured observational value of the Hubble constant analyzed by Planck. We shown that for the power-law terms the EoS parameter remains in quintessence and vacuum region, the stability condition is valid for all models. The preference of choosing this type of parameterized deceleration parameter is that it organize a wide class of feasible models of cosmic expansion. In cosmology, by choosing some particular evolution situation for a cosmological parameter and after that gauge the estimations 
Table 2 Results of the cosmological parameters, GSLT and thermal equilibrium condition

\begin{tabular}{|c|c|c|c|c|}
\hline Models of $\mathrm{H}(\mathrm{z})$ & $\omega_{e f f}$ & $v_{s}^{2}$ & GSLT & Thermal condition \\
\hline Model 1 & Quintessence region to vacuum region & Stable & Partially valid & Satisfy \\
\hline Model 2 & Quintessence region to vacuum region & Stable & Valid & Partially satisfy \\
\hline Model 3 & Quintessence region & Stable & Valid & Satisfy \\
\hline Model 4 ( $\Lambda$ CDM model) & Quintessence region to vacuum region & Stable & Valid & Partially satisfy \\
\hline
\end{tabular}

of the parameters with the support of various observational dataset, the parametric reconstruction procedure manages an attempt to develop a model. The plan to parameterize deceleration parameter $q(z)$ is a straightforward way to deal with the transition of the universe from decelerated expansion phase to accelerated expansion phase and furthermore establish possibilities for future examination in regards to the nature of the dark energy.

The results we analyzed from the values of the model parameter from $\mathrm{CC}+H_{o}$ dataset in $1 \sigma$ confidence level are given in Table 2.

Data Availability Statement This manuscript has no associated data or the data will not be deposited. [Authors' comment: All data generated or analysed during this study are included in this published article.]

Open Access This article is licensed under a Creative Commons Attribution 4.0 International License, which permits use, sharing, adaptation, distribution and reproduction in any medium or format, as long as you give appropriate credit to the original author(s) and the source, provide a link to the Creative Commons licence, and indicate if changes were made. The images or other third party material in this article are included in the article's Creative Commons licence, unless indicated otherwise in a credit line to the material. If material is not included in the article's Creative Commons licence and your intended use is not permitted by statutory regulation or exceeds the permitted use, you will need to obtain permission directly from the copyright holder. To view a copy of this licence, visit http://creativecomm ons.org/licenses/by/4.0/.

Funded by SCOAP ${ }^{3}$.

\section{References}

1. A.G. Riess et al., Astron. J. 116, 1009 (1998)

2. S. Perlmutter et al., Astrophys. J. 517, 565 (1999)

3. A.G. Riess et al., Astrophys. J. 607, 665 (2004)

4. M. Tegmark et al., Phys. Rev. D 69, 103501 (2004)

5. N. Benitez et al., Astrophys. J. 691, 241 (2009)

6. D. Parkinson et al., Mon. Not. R. Astron. Soc. 401, 2169 (2010)

7. D.N. Spergel et al., Astrophys. J. Suppl. 170, 377 (2007)

8. J. Dunkley et al., Astrophys. J. 701, 1804 (2009)

9. G. Hinshaw et al., Astrophys. J. 208, 19 (2013)

10. C.L. Bennett et al., Astrophys. J. 208, 20 (2013)

11. P.A.R. Ade et al., Astron. Astrophys. 571, A16 (2014)

12. P.A.R. Ade et al., Astron. Astrophys. 594, A13 (2016)

13. A.G. Riess et al., Astron. J. 116, 1009 (1998)

14. S. Perlmutter et al., Astrophys. J. 517, 565 (1999)

15. A.A. Mamon, S. Das, Int. Mod. Phys. J. D 25, 1650032 (2016)

16. S. Weinberg, Rev. Mod. Phys. 61, 1 (1989)

17. M.S. Turner, A.G. Riess, Astrophys. J. 569, 18 (2002)
18. Y.G. Gong, A. Wang, Phys. Rev. D 73, 083506 (2006)

19. Y.G. Gong, A. Wang, Phys. Rev. D 75, 043520 (2007)

20. L. Xu, H. Liu, Mod. Phys. Lett. A 23, 1939 (2008)

21. J.V. Cunha, Phys. Rev. D 79, 047301 (2009)

22. D.S. Campo et al., Phys. Rev. D 86, 083509 (2012)

23. B. Santos et al., Astropart. Phys. 35, 17 (2011)

24. A.A. Mamon, S. Das, Int. J. Mod. Phys. D. 25, 1650032 (2016)

25. A.A. Mamon, S. Das, Eur. Phys. J. C. 77, 495 (2017)

26. A.A. Mamon, Mod. Phys. Lett. A 33, 1850056 (2018)

27. H. Essen, Int. Theor. Phys. J. 29, 183-187 (1990)

28. V. Faraoni et al., Fund. Cosmic Phys. 20, 121 (1999)

29. P.D. Mannheim, Astrophys. J. 479, 659 (1997)

30. M.R. Tanhayi et al., Mod. Phys. Lett. A 26, 2403-2410 (2011)

31. Ghanaatian, M. et al., arXiv:1602.05077v1

32. G.U. Varieschi, K.L. Ault, Int. J. Mod. Phys. D 25, 1650064-15 (2016)

33. S.F. Wu et al., Nucl. Phys. B 799, 330-344 (2008)

34. A. Sheykhi et al., Nucl. Phys. B 779, 1-12 (2007)

35. A. Sheykhi et al., Phys. Rev. D 76, 023515 (2007)

36. R.G. Cai et al., Phys. Rev. D 78, 124012 (2008)

37. A. Paranjape et al., Phys. Rev. D 74, 104015 (2006)

38. S.F. Wu et al., Class. Quantum Gravity 25, 235018 (2008)

39. C. Eling et al., Phys. Rev. Lett. 96, 121301 (2006)

40. K. Bamba et al., Phys. Lett. B 688, 101-109 (2010)

41. M. Akbar, R.G. Cai, Phys. Lett. B 648, 243-248 (2007)

42. K. Bamba, C.Q. Geng, Phys. Lett. B 679, 282-287 (2009)

43. M. Akbar, R.G. Cai, Phys. Lett. B 635, 7-10 (2006)

44. R.G. Cai, L.M. Cao, Phys. Rev. D 75, 064008 (2007)

45. C.Q. Geng et al., Entropy 21, 172 (2019)

46. M. Zubair et al., Phys. Dark Univ. 14, 116-125 (2016)

47. M. Ghanaatian et al., Ann. Phys. 397, 458-473 (2018)

48. M.V. Takook, M.R. Tanhayi, High Energy Phys. 12, 1-15 (2010)

49. P.D. Mannheim, Phys. Rev. D 75, 124006 (2007)

50. O. Akarsu, T. Dereli, S. Kumar, L. Xu, Eur. Phys. J. 129, 22 (2014)

51. J.V. Cunha, J.A.S. Lima, Mon. Not. R. Astron. Soc. 390, 210-217 (2008)

52. B. Santos et al., Astropart. Phys. 35, 17-20 (2011)

53. R. Nair et al., JCAP 01, 018 (2012)

54. O. Akarsu et al., EPJ Plus 129, 22 (2014)

55. A. Sheykhi, Eur. Phys. J. C 69, 269 (2010)

56. M. Chavallier, D. Polarski, Int. J. Mod. Phys. D 10, 213 (2001)

57. E.V. Linder, Phys. Rev Lett. 90, 091301 (2003). (See also Barboza E.M. et al.: Phys. Rev. D 80, 043521 (2009))

58. J. Sollerman et al., Astrophys. J. 703, 1374 (2009)

59. M. Sharif, A. Ikram, Adv. High Energy Phys. 2018, 2563871 (2018)

60. S.W. Hawking, Commun. Math. Phys. 43, 199 (1975)

61. J.D. Bekenstein, Phys. Rev. D 7, 2333 (1973)

62. J.M. Bardeen, B. Carter, S.W. Hawking, Community Math. Phys. 31, 161 (1973)

63. J.D. Bekenstein, Lett. Nuovo Cim. 4, 740 (1972)

64. R.G. Cai, S.P. Kim, JHEP 02, 050 (2005)

65. R. Jimenez, A. Loeb, Astrophys. J. 37, 573 (2002)

66. S. Perlmutter et al., Astrophys. J. 517, 565-586 (1999) 